\title{
CONSTRAINTS ON DISTRIBUTIONS IMPOSED BY PROPERTIES OF LINEAR FORMS*
}

\author{
Denis Belomestny ${ }^{1}$
}

\begin{abstract}
Let $\left(X_{1}, Y_{1}\right), \ldots,\left(X_{m}, Y_{m}\right)$ be $m$ independent identically distributed bivariate vectors and $L_{1}=\beta_{1} X_{1}+\ldots+\beta_{m} X_{m}, L_{2}=\beta_{1} Y_{1}+\ldots+\beta_{m} Y_{m}$ are two linear forms with positive coefficients. We study two problems: under what conditions does the equidistribution of $L_{1}$ and $L_{2}$ imply the same property for $X_{1}$ and $Y_{1}$, and under what conditions does the independence of $L_{1}$ and $L_{2}$ entail independence of $X_{1}$ and $Y_{1}$ ? Some analytical sufficient conditions are obtained and it is shown that in general they can not be weakened.
\end{abstract}

Mathematics Subject Classification. 62E10, 60E10.

Received April 22, 2002. Revised March 17, 2003.

\section{INTRODUCTION}

While testing homogeneity or independence hypothesis it occurs sometimes that we have access to the transformed random sample rather than to the original one. Scaling and superposition are among the most common transformations. Typical examples are the mixtures of simultaneous speech signals that have been picked up by several microphones, interfering radio signals arriving at a mobile phone, or parallel time series obtained from some industrial process. Specifically, we consider here the following two setups and two corresponding problems.

- Let us have two vectors of independent and identically distributed random variables $\mathbf{X}=\left(X_{1}, \ldots X_{m}\right)$ and $\mathbf{Y}=\left(Y_{1}, \ldots, Y_{m}\right)$. The problem consists in testing the hypothesis that the distributions of $\mathbf{X}$ and $\mathbf{Y}$ are equal on the basis of two linear statistics:

$$
\begin{aligned}
& L_{\mathbf{X}}=\beta_{1} X_{1}+\ldots+\beta_{m} X_{m}, \\
& L_{\mathbf{Y}}=\beta_{1} Y_{1}+\ldots+\beta_{m} Y_{m},
\end{aligned}
$$

where $\left\{\beta_{i}\right\}$ are some positive real numbers.

- Let us have $m$ independent identically distributed bivariate vectors

$$
\left(X_{1}, Y_{1}\right),\left(X_{2}, Y_{2}\right), \ldots,\left(X_{m}, Y_{m}\right)
$$

Keywords and phrases. Equidistribution, independence, linear forms, characteristic functions.

* Research is partly supported by Russian Foundation of Fundamental Research (Grant No. 00-01-00661).

1 Institute fur Angewandte Mathematik, Universität Bonn, Interdisziplinares Zentrum für Komplexe Systeme,

Meckenheimer Allee 176, 53115 Bonn, Germany; e-mail: db@izks.uni-bonn.de 
The matter in question is how one can test the independence of $\mathbf{X}$ and $\mathbf{Y}$ having at hand $L_{\mathbf{X}}$ and $L_{\mathbf{Y}}$ only.

Regardless of the type of statistical inferences, the corresponding identifiability problems are of paramount importance.

The problem of defining the distribution of $\mathbf{X}$ by means of $L_{\mathbf{X}}$ for the case when all $\left\{\beta_{k}\right\}$ are equal has been considered in $[1]$ and $[8]$. We mention also that the first problem considered in our paper is different from the phenomena investigated by Marcinkiewicz in 1938 (see [6]) and later by Linnik (see [5]). They dealt with two identically distributed different linear forms in the same i.i.d. variables and tried to get the necessary and sufficient conditions for the Gaussianity. We investigate the same identically distributed linear forms in possibly different i.i.d. random variables and are interested in the conditions for the equidistribution of these variables.

\section{The Main Results}

Let us rewrite our linear statistics in the following form

$$
\begin{aligned}
& L_{\mathbf{X}}=b_{1}\left(X_{11}+\ldots+X_{1 k_{1}}\right)+\ldots+b_{n}\left(X_{n 1}+\ldots+X_{n k_{n}}\right), \\
& L_{\mathbf{Y}}=b_{1}\left(Y_{11}+\ldots+Y_{1 k_{1}}\right)+\ldots+b_{n}\left(Y_{n 1}+\ldots+Y_{n k_{n}}\right),
\end{aligned}
$$

where $\left\{k_{i}\right\}$ are natural numbers with $k_{1}+\ldots+k_{n}=m$ and $\left\{b_{i}\right\}$ satisfy the condition

$$
b_{1}<\ldots<b_{n}
$$

If $n>1$, we set

$$
\begin{gathered}
q_{i}=\frac{k_{i}}{k_{n}}, h_{i}=\frac{b_{i}}{b_{n}}, \quad i=1, \ldots, n-1, \\
\tau(z)=1+q_{1} h_{1}^{z}+\ldots+q_{n-1} h_{n-1}^{z} .
\end{gathered}
$$

By $F_{1}(x), f_{1}(t)$ and $F_{2}(x), f_{2}(t)$ we also denote the common distribution and the characteristic functions corresponding to the samples $\mathbf{X}$ and $\mathbf{Y}$ and by $F(x, y), f(x, y)$ the common distribution and the characteristic functions of the pairs $\left\{\left(X_{i}, Y_{i}\right), i=1, \ldots, m\right\}$. Throughout the paper $\cong$ will stand for equality in law.

Theorem 1.1. I) Let two random samples $\mathbf{X}$ and $\mathbf{Y}$ be such that $L_{\mathbf{X}} \cong L_{\mathbf{Y}}$.

If one of the following conditions is fulfilled

(1) $n=1$;

(2) $n>1, q_{1}+\ldots+q_{n-1} \leq 1$;

(3) $n>1, q_{1}+\ldots+q_{n-1}>1$ and $F_{1}(x)$ possesses finite absolute moments of the order $\alpha$, where $\alpha=$ $\max \{\Re z: \tau(z)=0\}>0$,

then $f_{1}(t)=f_{2}(t)$ for $|t|<\delta$, where $\delta=\min \left\{t>0:\left|f_{1}(t)\right|=0\right\}$.

II) If conditions $(1,2)$ and (3) are violated then there exist two distribution functions $F_{10}(x)$ and $F_{20}(x)$ possessing absolute moments of the order $\alpha-\epsilon$ for any $\epsilon>0$ such that $L_{\mathbf{X}_{\mathbf{0}}} \cong L_{\mathbf{Y}_{\mathbf{0}}}$ and at the same time $f_{10}\left(t_{n}\right) \neq f_{20}\left(t_{n}\right)$ for some sequence $t_{n}$ tending to 0 .

Corollary 1.2. Let the conditions of Theorem 1.1 be fulfilled. If $f_{1}(t)$ does not vanish on $\mathbb{R}$, then $F_{1}(x) \equiv F_{2}(x)$.

Corollary 1.3. Let the conditions of Theorem 1.1 be satisfied. If $f_{1}(t)$ has an unique extension from any interval containing zero on $\mathbb{R}$ (see [2]), then $F_{1}(x) \equiv F_{2}(x)$. 
Theorem 1.4. I) Let two random sample $\mathbf{X}$ and $\mathbf{Y}$ be such that $L_{\mathbf{X}}$ and $L_{\mathbf{Y}}$ are independent. If one of the following conditions is fulfilled

(1) $n=1$;

(2) $n>1, q_{1}+\ldots+q_{n-1} \leq 1$;

(3) $n>1, q_{1}+\ldots+q_{n-1}>1$ and $F(x, y)$ possesses all absolute moments of the order $\alpha$ :

$$
\int_{-\infty}^{\infty}|x|^{\alpha_{1}}|y|^{\alpha_{2}} \mathrm{~d} F(x, y)<\infty, \quad \alpha_{1}+\alpha_{2}=\alpha, \quad \alpha_{1}, \alpha_{2}>0
$$

where $\alpha=\max \{\Re z: \tau(z)=0\}>0$,

then $f(t, s)=f_{1}(t) f_{2}(s)$ for $\max \{|t|,|s|\}<\delta$, where $\delta=\min \{t, s>0:|f(t, s)|=0\}$.

II) If conditions (1, 2) and (3) are violated, then there exists a distribution function $F_{0}(x, y)$ possessing all absolute moments of the order $\alpha-\epsilon$ for any $\epsilon>0$ such that $L_{\mathbf{X}_{\mathbf{0}}}$ and $L_{\mathbf{Y}_{\mathbf{0}}}$ are independent, but $f_{0}\left(t_{n}, s_{n}\right) \neq$ $f_{10}\left(t_{n}\right) f_{20}\left(s_{n}\right)$ for some sequences $t_{n}$ and $s_{n}$ tending to zero.

Remark 1.5. The condition (4) is equivalent to the following one

$$
\int_{-\infty}^{\infty}\left(|x|^{\alpha}+|y|^{\alpha}\right) \mathrm{d} F(x, y)<\infty
$$

Corollary 1.6. Let the conditions of Theorem 1.4 be fulfilled. If $f(t, s)$ does not vanish on $\mathbb{R}^{2}$, then $F(x, y) \equiv$ $F_{1}(x) F_{2}(y)$.

Corollary 1.7. Let the conditions of Theorem 1.4 be fulfilled. If $f(t, s)$ has an unique extension from any square with the centre at zero on $\mathbb{R}^{2}$, then $F(x, y) \equiv F_{1}(x) F_{2}(y)$.

\section{Auxiliary Results}

Lemma 2.1. Let $X_{1}, \ldots, X_{n}$ be independent identically distributed random variables and

$$
L=a_{1} X_{1}+\ldots+a_{n} X_{n}
$$

where $a_{1}, \ldots, a_{n}$ are arbitrary finite real numbers. Then for any $r>0$, the condition $\mathbf{E}|L|^{r}<\infty$ is equivalent to $\mathbf{E}\left|X_{1}\right|^{r}<\infty$.

Proof. First of all we prove that

$$
\mathbf{E}|L|^{r}<\infty \Longrightarrow \mathbf{E}\left|X_{1}\right|^{r}<\infty
$$

Without loss of generality we assume that $a_{1} \neq 0$. Let us set $b=-m\left(a_{2} X_{2}+\ldots+a_{n} X_{n}\right)$, then

$$
\left|a_{1} X_{1}-b\right| \leq \max _{1 \leq k \leq n}\left|S_{k}-m\left(S_{k}-S_{n}\right)\right|
$$

where

$$
S_{k}=a_{1} X_{1}+\ldots+a_{k} X_{k}, \quad k=1, \ldots, n .
$$

According to Levy inequality ([7], p. 51)

$$
\operatorname{Pr}\left[\left|a_{1} X_{1}-b\right| \geq x\right] \leq \operatorname{Pr}\left[\max _{1 \leq k \leq n}\left|S_{k}-m\left(S_{k}-S_{n}\right)\right|\right] \leq 2 \operatorname{Pr}\left[\left|S_{n}\right| \geq x\right]
$$

that implies

$$
\mathbf{E}\left|a_{1} X_{1}-b\right|^{r} \leq 2 \mathbf{E}\left|S_{n}\right|^{r}=2 \mathbf{E}|L|^{r}
$$


Further, if $r \leq 1$

$$
\mathbf{E}\left|a_{1} X_{1}\right|^{r} \leq \mathbf{E}\left|a_{1} X_{1}-b\right|^{r}+|b|^{r}<\infty,
$$

and if $r>1$

$$
\mathbf{E}\left|a_{1} X_{1}\right|^{r} \leq 2^{r-1} \mathbf{E}\left|a_{1} X_{1}-b\right|^{r}+2^{r-1}|b|^{r}<\infty .
$$

In order to prove the inverse statement it is enough to notice that

$$
\mathbf{E}|L|^{r} \leq\left|a_{1}\right|^{r} \mathbf{E}\left|X_{1}\right|^{r}+\ldots+\left|a_{n}\right|^{r} \mathbf{E}\left|X_{n}\right|^{r}
$$

for $r \leq 1$ and

$$
\mathbf{E}|L|^{r} \leq n^{r}\left(\left|a_{1}\right|^{r} \mathbf{E}\left|X_{1}\right|^{r}+\ldots+\left|a_{n}\right|^{r} \mathbf{E}\left|X_{n}\right|^{r}\right)
$$

for $r>1$.

Lemma 2.2. Let $X$ and $Y$ be two random variables with the characteristic functions $f(t)$ and $g(t)$. If for some natural number $m, \mathbf{E}|X|^{m}<\infty, \mathbf{E}|Y|^{m}<\infty$ and there exists tending to zero sequence $t_{n}$ such that

$$
f\left(t_{n}\right)=g\left(t_{n}\right), \quad n \in \mathbb{N},
$$

then

$$
f^{(l)}(0)=g^{(l)}(0), \quad l=0, \ldots, m .
$$

Proof. We notice that $f(t)$ and $g(t)$ are $m$ times continuously differentiable on the real line. Applying consecutively the Rolle theorem to the functions

$$
f^{(l)}(t)-g^{(l)}(t), \quad l=0, \ldots, m
$$

and taking into account (5), we can find tending to zero sequences $\left\{t_{l, n}, l=1, \ldots, m, n \in \mathbb{N}\right\}$ such that

$$
f^{(l)}\left(t_{l, n}\right)=g^{(l)}\left(t_{l, n}\right), \quad n \in \mathbb{N} .
$$

Lemma 2.3. For any characteristic function $f(t)$ the following inequalities hold

$$
\begin{gathered}
|\Im f(t)| \leq \sqrt{2(1-\Re f(t))}, \\
|\Im f(a t)+\Im f(b t)+\Im f(-(a+b) t)| \leq(1-\Re f(a t))+(1-\Re f(b t))+(1-\Re f(-(a+b) t)), \quad a, b \in \mathbb{R} .
\end{gathered}
$$

Proof. The proof of the first inequality can be found in [9] (Chap. 2), of the second one in [5] (p. 230).

Lemma 2.4. A distribution $F(x)$ with characteristic function $f(t)$ has finite absolute moment of $2 k+\lambda$ order, where $k \in \mathbb{N}, 0<\lambda<2$ if and only if for any finite $\delta>0$

$$
\int_{0}^{\delta} t^{-\lambda-1}\left(1-\Re \frac{f^{(2 k)}(t)}{f^{(2 k)}(0)}\right) \mathrm{d} t<\infty .
$$

Proof. Can be found in [3].

Proposition 2.5. Let continuous and bounded function $w(x)$ satisfy the equation

$$
q_{1} w\left(x+\gamma_{1}\right)+\ldots+q_{m} w\left(x+\gamma_{m}\right)+w(x)=0, \quad x>0
$$

where $q_{i}$ and $\gamma_{i}$ are positive numbers. Set

$$
\alpha=\min \{\Re z: \tau(z)=0\},
$$


where $\tau(z)=1+q_{1} \mathrm{e}^{\gamma_{1} z}+\ldots+q_{m} \mathrm{e}^{\gamma_{m} z}$. If one of the following conditions is fulfilled,

(1) $q_{1}+\ldots+q_{m}<1$;

(2) $q_{1}+\ldots+q_{m}=1, \lim _{x \rightarrow \infty}|w(x)|=0$;

(3) $\int_{0}^{\infty} \mathrm{e}^{-\alpha x}|w(x)| \mathrm{d} x<\infty$,

then $w(x) \equiv 0$ for $x>0$.

Proof. If $q_{1}+\ldots+q_{m} \leq 1$ then (6) after $n$ iterations yields

$$
w(x)=(-1)^{n} \sum_{n_{1}+\ldots+n_{m}=n} \frac{n !}{n_{1} ! \ldots n_{m} !} q_{1}^{n_{1}} \cdot \ldots \cdot q_{m}^{n_{m}} w\left(x+n_{1} \gamma_{1}+\ldots+n_{m} \gamma_{m}\right)
$$

hence

$$
|w(x)| \leq\left(q_{1}+\ldots+q_{m}\right)^{n} \max _{\theta>n \gamma_{*}}|w(x+\theta)| \rightarrow 0, \quad n \rightarrow \infty,
$$

where $\gamma_{*}=\min \left\{\gamma_{1}, \ldots, \gamma_{m}\right\}>0$.

Let condition (3) be fulfilled. Applying the Laplace transform to (6), we have

$$
\Phi(z) \tau(z)-M(z)=0
$$

where

and

$$
\Phi(z)=\int_{0}^{\infty} \mathrm{e}^{-z x} w(x) \mathrm{d} x
$$

$$
M(z)=\sum_{k=1}^{m} q_{k} \mathrm{e}^{\gamma_{k} z} \int_{0}^{\gamma_{k}} \mathrm{e}^{-z x} w(x) \mathrm{d} x .
$$

Lemma 2.6. $\quad$ 1. $\Phi(z)$ is analytic in the domain $\{\Re z \geq \alpha\}$ and can be expressed there in the form

$$
\Phi(z)=\frac{M(z)}{\tau(z)}
$$

2. $M(z)$ is an entire function bounded by a constant $B=B(c)$ in every half-plane $\Re z \leq c$.

3. The function $\tau(z)$ has the following properties:

(a) $\tau(z)$ is an entire function, almost periodic on every line $\Re z=$ const;

(b) $\tau(z)$ does not have zeros for $\Re z<\alpha$;

(c) the number of zeros of $\tau(z)$ in any closed rectangle $\{a \leq \Re z \leq b, y \leq \Im z \leq y+1\}$ is bounded above by the number $N(a, b)$, the latter being independent of $y$;

(d) if the distance between some point $z_{0}$ and every zero of $\tau(z)$ is larger than $\epsilon>0$, then $\left|\tau\left(z_{0}\right)\right|>A(\epsilon)$.

Proof of Lemma 2.6. (1) follows from condition (2) of Proposition 2.5 and (7, 2) and (3)(a) arise from the definition, (3)(b), (3)(d) are corollaries of the properties of almost-periodic functions and are proved, for example, in [4] (Chap. 6, Sect. 2).

Corollary 2.7. For any $c>0$ there exists sequence $T_{k} \rightarrow \infty$ such that $|\tau(z)|>A$ for $|\Im z|=T_{k}, k \in \mathbb{N}, \Re z<c$.

Proof of Corollary 2.7. It follows from (3)(c) that for any natural number $k$ there exists $\epsilon>0$, not depending on $k$ and $T_{k} \in[k, k+1]$ such that all points of the segment $\left\{\alpha \leq \Re z \leq c, \Im z=T_{k}\right\}$ are at a distance greater than $\epsilon$ from the zeros of $\tau(z)$. Since $\tau(z) \neq 0$ for $\Re z<\alpha$, the same holds for the half-line $\Re z \leq c$. According to $(3)(\mathrm{d}),|\tau(z)|>A=A(\epsilon)$ for all $z$ from $\left\{\Re z \leq c, \Im z=T_{k}\right\}$. All zeros of $\tau(z)$ can be split into pairs of complex conjugates and therefore the same is true for the half-lines $\left\{\Re z \leq c, \Im z=-T_{k}\right\}$. 
Using the formula of complex inversion for the Laplace transform, we have for $u>0$

$$
\int_{0}^{u} w(x) \mathrm{d} x=\lim _{T \rightarrow \infty} \frac{1}{2 \pi i} \int_{c-i T}^{c+i T} \frac{\mathrm{e}^{u z} \Phi(z)}{z} \mathrm{~d} z, \quad c>0 .
$$

The function $\frac{M(z)}{\tau(z)}$ is an entire function that is equal to $\Phi(z)$ when $\Re z>\alpha$. Let $h<\alpha<0$, then, according to the Cauchy theorem,

$$
\frac{1}{2 \pi i} \int_{c-i T}^{c+i T} \frac{\mathrm{e}^{u z} M(z)}{z \tau(z)} \mathrm{d} z=\frac{1}{2 \pi i}\left[\int_{h+i T}^{c+i T}-\int_{h-i T}^{c-i T}+\int_{h-i T}^{h+i T} \frac{\mathrm{e}^{u z} M(z)}{z \tau(z)} \mathrm{d} z\right]+\Phi(0) .
$$

Further, for any fixed $k$

$$
\left|\int_{h-i T_{k}}^{h+i T_{k}} \frac{\mathrm{e}^{u z} M(z)}{z \tau(z)} \mathrm{d} z\right| \leq \frac{2(k+1) B}{|h| A(|h|-|\alpha|)} \mathrm{e}^{h u} \rightarrow 0, h \rightarrow-\infty
$$

and

$$
\left|\int_{-\infty+i T_{k}}^{c+i T_{k}}-\int_{-\infty-i T_{k}}^{c-i T_{k}} \frac{\mathrm{e}^{u z} M(z)}{z \tau(z)} \mathrm{d} z\right| \leq \frac{B}{A} \mathrm{e}^{u c} \frac{2}{\sqrt{c^{2}+T_{k}^{2}}} \rightarrow 0, k \rightarrow \infty .
$$

Taking into account that $\Phi(0)=\int_{0}^{\infty} w(x) \mathrm{d} x$, we finally get

$$
\int_{u}^{\infty} w(x) \mathrm{d} x=0, \quad u>0 .
$$

Lemma 2.8. Let $0<\mu \leq 2, \lambda \geq \mu, \beta$ be real numbers, then there exists a positive number $A$, a nonnegative number $D$ and a natural number $m \leq 2$ such that the function

$$
f(t)=\exp \left[-|t|^{\mu}-A|t|^{\lambda}\left(1+B \mathrm{e}^{i \frac{t}{|t|} \beta \ln (|t|)}+D \ln ^{m}|t|\right)\right]
$$

is a characteristic function for any $B$ that satisfies the condition

$$
|B|<B(\lambda, \beta)=\left\{\begin{array}{r}
|\sin (\lambda \pi / 2)| \exp (-\beta \pi / 2), \lambda \neq 2 k, k \in \mathbb{N} \\
\exp (-\beta \pi / 2), \lambda=2 k, k \in \mathbb{N} .
\end{array}\right.
$$

Proof. Let first $A, B$ and $D$ be less than 1 in absolute value. By virtue of the summability and the Hermitiance of $f(t)$ it is enough to prove that

$$
p(x)=\frac{1}{\pi} \Re \int_{0}^{\infty} \mathrm{e}^{-i t x} f(t) \mathrm{d} t \geq 0, \quad x \in \mathbb{R} .
$$

Now in complex plane $z=t+i s$ we consider the rectangular contour generated by the intersection of the coordinate axes and two lines $s=-S, t=T$. Since $f(z)$ is analytic in the domain $\{\Re z>0, \Im z<0\}$ and continuous on its boundary, the Cauchy theorem implies

$$
\int_{0}^{T} \mathrm{e}^{-i t x} f(t) \mathrm{d} t=-i \int_{0}^{S} \mathrm{e}^{-s x} f(-i s) \mathrm{d} s+\int_{0}^{T} \mathrm{e}^{-i(t-i S) x} f(t-i S) \mathrm{d} t+i \int_{0}^{S} \mathrm{e}^{-i(T-i s) x} f(T-i s) \mathrm{d} s .
$$

It is easy to check that uniformly in $s$

$$
|f(T-i s)|=O\left(\mathrm{e}^{-T^{\mu}}\right), \quad T \rightarrow \infty .
$$


Therefore, the third integral in (9) converges to 0 when $T \rightarrow \infty$ and the second one does not exceed in absolute value

$$
\mathrm{e}^{-S x} \int_{0}^{T}|f(t-i S)| \mathrm{d} t=O\left(\mathrm{e}^{-S x}\right)
$$

Turn now to the first integral in (9). Since

$$
-i \int_{0}^{S} \mathrm{e}^{-s x} f(-i s) \mathrm{d} s=-i \int_{0}^{\infty} \mathrm{e}^{-s x} f(-i s) \mathrm{d} s+i \int_{S}^{\infty} \mathrm{e}^{-s x} f(-i s) \mathrm{d} s
$$

and

$$
\left|\int_{S}^{\infty} \mathrm{e}^{-s x} f(-i s) \mathrm{d} s\right| \leq \mathrm{e}^{-S x / 2} \int_{S}^{\infty} \mathrm{e}^{-s x / 2}|f(-i s)| \mathrm{d} s \leq C \mathrm{e}^{-S x / 2},
$$

we have with regard to $(9)$

$$
\Re \int_{0}^{\infty} \mathrm{e}^{-i t x} f(t) \mathrm{d} t=\Re\left(-i \int_{0}^{\infty} \mathrm{e}^{-s x} f(-i s) \mathrm{d} s\right)+R(x)
$$

where

$$
|R(x)| \leq C \mathrm{e}^{-S x / 2} .
$$

Further, it can be shown that there exists a real number $X$ not depending on $\lambda, \mu, A, B$, such that for sufficiently large $S, x>X$ and for $B$ satisfying (8), the right-hand side of (10) is nonnegative.

Let now $x \leq X$. We have

$$
\begin{aligned}
& \int_{0}^{\infty} \mathrm{e}^{-i t x} \exp \left[-t^{\mu}\right.\left.-A t^{\lambda}\left(1+B \mathrm{e}^{i \beta \ln (t)}+D \ln ^{m}(t)\right)\right]=\int_{0}^{\infty} \exp \left(-i t x-t^{\mu}\right) \mathrm{d} t \\
&+ \int_{0}^{T} \exp \left(-i t x-t^{\mu}\right)\left(\exp \left[-A t^{\lambda}\left(1+B \mathrm{e}^{i \beta \ln (t)}+D \ln ^{m}(t)\right)\right]-1\right) \mathrm{d} t \\
&+ \int_{T}^{\infty} \exp \left(-i t x-t^{\mu}\right)\left(\exp \left[-A t^{\lambda}\left(1+B \mathrm{e}^{i \beta \ln (t)}+D \ln ^{m}(t)\right)\right]-1\right) \mathrm{d} t \\
&=I_{1}+I_{2}+I_{3}
\end{aligned}
$$

Using the inequality $\left|\mathrm{e}^{z}-1\right| \leq|z| \mathrm{e}^{|z|}$ that holds for any complex $z$, we obtain

$$
\left|I_{2}\right| \leq \int_{0}^{T} \exp \left(-t^{\mu}\right) \exp \left(A t^{\lambda}\left(2+\ln ^{2}(t)\right) A t^{\lambda}\left(2+\ln ^{2}(t)\right) \mathrm{d} t \leq A C_{4} \exp \left(A T^{\lambda}\left(2+\ln ^{2}(T)\right)\right.\right.
$$

and

$$
\left|I_{3}\right| \leq C_{5} \mathrm{e}^{-T^{\mu / 2}} .
$$

Making use of the fact that $\Re I_{1}(x)$ is positive function on the real line, one get $\Re I_{1}(x)>\delta>0$ for $x \leq X$. Picking up $T_{0}$ and $A$ in a proper way, we conclude that the left-hand side (11) is positive.

Lemma 2.9. Let $0<\mu \leq 2, \lambda \geq \mu, \beta$ be some real numbers. Then there exist positive numbers $A_{1}, A_{2}, B$, nonnegative numbers $D_{1}, D_{2}$ and a natural number $m \leq 2$ such that function

$$
\begin{aligned}
f(t, s)=\exp \left[-|t|^{\mu}-A_{1}|t|^{\lambda}\left(1+D_{1} \ln ^{m}|t|\right)-|s|^{\mu}-A_{2}|s|^{\lambda}\left(1+D_{2} \ln ^{m}|s|\right)\right. & \left.-B|t|^{\lambda / 2}|s|^{\lambda / 2} \mathrm{e}^{i \frac{t}{2|t|} \beta \ln (|t|)} \mathrm{e}^{i \frac{s}{2|s|} \beta \ln (|s|)}\right]
\end{aligned}
$$

is a characteristic function of some bivariate distribution. 
Proof. Is analogous to the one of Lemma 2.8 .

\section{Proof of the MAin Results}

Theorem 3.1. Let $f(t)$ and $g(t)$ be two characteristic functions satisfying the equation

$$
f^{k_{1}}\left(b_{1} t\right) \cdot \ldots \cdot f^{k_{n}}\left(b_{n} t\right)=g^{k_{1}}\left(b_{1} t\right) \cdot \ldots \cdot g^{k_{n}}\left(b_{n} t\right), \quad t \in \mathbb{R} .
$$

If one of the following conditions is fulfilled

1. $n=1$;

2. $n>1, q_{1}+\ldots+q_{n-1} \leq 1$;

3. $n>1, q_{1}+\ldots+q_{n-1}>1$ and distribution function $F(x)$ corresponding to $f(t)$ possesses finite moment of the order $\alpha$, where $\alpha=\max \{\Re z: \tau(z)=0\}$,

then $f(t)=g(t)$ for $|t|<\delta$, where $\delta=\min \{t>0:|f(t)|=0\}$.

Proof. We see from (12) that $g(t) \neq 0$ for $|t|<\delta$ and therefore one can define the following functions

$$
\zeta(t)=\ln f(t), \xi(t)=\ln g(t), \quad|t|<\delta .
$$

It follows from (12) that

$$
k_{1} \zeta\left(b_{1} t\right)+\ldots+k_{n} \zeta\left(b_{n} t\right)=k_{1} \xi\left(b_{1} t\right)+\ldots+k_{n} \xi\left(b_{n} t\right), \quad|t|<\delta / b_{n} .
$$

Further, designating

$$
\Delta(t)=\zeta(t)-\xi(t)
$$

we have

If $n=1$, then

$$
k_{1} \Delta\left(b_{1} t\right)+\ldots+k_{n} \Delta\left(b_{n} t\right)=0, \quad|t|<\delta / b_{n} .
$$

$$
k_{1} \Delta(t)=0, \quad|t|<\delta
$$

and Theorem 3.1 is proved. Let now $n>1$ then with notations (2) the equation (13) can be rewritten in the following way

$$
q_{1} \Delta\left(h_{1} t\right)+\ldots+q_{n-1} \Delta\left(h_{n-1} t\right)+\Delta(t)=0, \quad|t|<\delta .
$$

Setting

we get from (14)

$$
S(u)=\Delta\left(\mathrm{e}^{-u}\right)
$$

$$
q_{1} S\left(u+\gamma_{1}\right)+\ldots+q_{n-1} S\left(u+\gamma_{n-1}\right)+S(u)=0, \quad u \in(-\ln (\delta), \infty) .
$$

If $q_{1}+\ldots+q_{n-1} \leq 1$ then due to Proposition $2.5 S(u)=0$ for $u>-\ln (\delta)$ and hence $f(t) \equiv g(t)$ on $(-\delta, \delta)$.

Let us turn now to the case $q_{1}+\ldots+q_{n-1}>1$. We put

$$
\begin{aligned}
& \Delta_{M}(t)=\Re \Delta(t)=\ln |f(t)|-\ln |g(t)|, \\
& \Delta_{A}(t)=\Im \Delta(t)=\arg f(t)-\arg g(t) .
\end{aligned}
$$

The equation (14) is split now into two

$$
\begin{gathered}
q_{1} \Delta_{M}\left(h_{1} t\right)+\ldots+q_{n-1} \Delta_{M}\left(h_{n-1} t\right)+\Delta_{M}(t)=0, \quad|t|<\delta \\
q_{1} \Delta_{A}\left(h_{1} t\right)+\ldots+q_{n-1} \Delta_{A}\left(h_{n-1} t\right)+\Delta_{A}(t)=0, \quad|t|<\delta .
\end{gathered}
$$


Lemma 3.2. There exist sequences $t_{n}$ and $s_{n}$ tending to 0 as $n \rightarrow \infty$ such that

$$
\Delta_{M}\left(t_{n}\right)=0, \quad \Delta_{A}\left(s_{n}\right)=0, \quad n \in \mathbb{N}
$$

Proof. Let us introduce the number $\lambda$ as the real root of the equation

$$
q_{1} h_{1}^{\lambda}+\ldots+q_{n-1} h_{n-1}^{\lambda}=1
$$

then defining for $t>0$ the functions

$$
\Delta_{M}^{\lambda}(t)=\frac{\Delta_{M}(t)}{t^{\lambda}}, \Delta_{A}^{\lambda}(t)=\frac{\Delta_{A}(t)}{t^{\lambda}}
$$

one get

$$
\begin{aligned}
& p_{1} \Delta_{M}^{\lambda}\left(h_{1} t\right)+\ldots+p_{n-1} \Delta_{M}^{\lambda}\left(h_{n-1} t\right)+\Delta_{M}^{\lambda}(t)=0, \quad 0<t<\delta \\
& p_{1} \Delta_{A}^{\lambda}\left(h_{1} t\right)+\ldots+p_{n-1} \Delta_{A}^{\lambda}\left(h_{n-1} t\right)+\Delta_{A}^{\lambda}(t)=0, \quad 0<t<\delta
\end{aligned}
$$

where $\sum_{k=1}^{n-1} p_{k}=1$. Next, let us apply the first mean value theorem to (17) and (18)

$$
\Delta_{M}^{\lambda}\left(h_{M}(t) t\right)+\Delta_{M}^{\lambda}(t)=0, \Delta_{A}^{\lambda}\left(h_{A}(t) t\right)+\Delta_{A}^{\lambda}(t)=0, \quad t \in(0, \delta)
$$

where $h_{1} \leq h_{A}(t) \leq h_{n-1}$ and $h_{1} \leq h_{M}(t) \leq h_{n-1}$. For any $t>0$ there exist two sequences $a_{k}=a_{k}(t)$ and $b_{k}=b_{k}(t)$ such that

and

$$
h_{1}^{k} \leq a_{k}(t) \leq h_{n-1}^{k}, h_{1}^{k} \leq b_{k}(t) \leq h_{n-1}^{k}
$$

$$
\Delta_{M}^{\lambda}\left(a_{k}(t) t\right)=(-1)^{k} \Delta_{M}^{\lambda}(t), \Delta_{A}^{\lambda}\left(b_{k}(t) t\right)=(-1)^{k} \Delta_{A}^{\lambda}(t), \quad t \in(0, \delta)
$$

for $k \in \mathbb{N}$. Due to the continuity of $\Delta_{A}^{\lambda}(t)$ and $\Delta_{M}^{\lambda}(t)$ we conclude that there exist two sequences $t_{k} \rightarrow 0$ and $s_{k} \rightarrow 0$ such that

$$
\Delta_{M}^{\lambda}\left(t_{k}\right)=0, \Delta_{A}^{\lambda}\left(s_{k}\right)=0, \quad k \in \mathbb{N}
$$

Recalling the definition of $\Delta_{M}^{\lambda}(t)$ and $\Delta_{A}^{\lambda}(t)$, and the fact that $\Delta_{M}(0)=\Delta_{A}(0)=0$ we complete the proof.

Let us consider two symmetric characteristic functions $\phi(t)=|f(t)|^{2}$ and $\psi(t)=|g(t)|^{2}$ corresponding to the distribution functions $F(x) *(1-F(-x-0))$ and $G(x) *(1-G(-x-0))$. According to Lemma 2.1 these distributions have finite absolute moments of $\alpha$ order. We can represent the number $\alpha$ in the form $2 k+\lambda$, where $0<\lambda<2$. Now Lemma 2.2 implies

$$
\phi^{(l)}(0)=\psi^{(l)}(0), \quad l=0, \ldots, 2 k .
$$

Further,

$$
\frac{\Delta_{M}(t)}{2}=\ln \phi(t)-\ln \psi(t)=\ln \left(1+\frac{\phi(t)-\psi(t)}{\psi(t)}\right)
$$

and due to the continuity of $\phi(t)$ and $\psi(t)$, we can find number $\delta>\delta_{0}>0$ such that

$$
\left|\frac{\phi(t)-\psi(t)}{\psi(t)}\right|<\frac{1}{4}, \quad \psi(t)>\frac{2}{3}
$$

for $|t|<\delta_{0}$. Using the inequality

$$
2 / 3|x| \leq|\ln (1-x)|, \quad|x|<1 / 4
$$


we have for $|t|<\delta_{0}$

$$
\left|\Delta_{M}(t)\right| \leq \frac{4}{3}\left|\frac{\phi(t)-\psi(t)}{\psi(t)}\right| \leq 2|\phi(t)-\psi(t)| .
$$

Upon expanding the function $\phi(t)-\psi(t)$ into Taylor series with remainder term in an integral form and taking (19) into account, one has

$$
\phi(t)-\psi(t)=\frac{1}{(2 k-1) !} \int_{0}^{t} \phi^{(2 k)}(t)(t-u)^{2 k-1} \mathrm{~d} u-\frac{1}{(2 k-1) !} \int_{0}^{t} \psi^{(2 k)}(t)(t-u)^{2 k-1} \mathrm{~d} u .
$$

Since $\phi^{(2 k)}(0)=\psi^{(2 k)}(0), \phi^{(2 k)}(0)>\phi^{(2 k)}(t)$ and $\psi^{(2 k)}(0)>\psi^{(2 k)}(t)$,

$$
\begin{aligned}
|\phi(t)-\psi(t)| \leq & \frac{1}{(2 k-1) !} \int_{0}^{t}\left(\phi^{(2 k)}(0)-\phi^{(2 k)}(t)\right)(t-u)^{2 k-1} \mathrm{~d} u \\
& +\frac{1}{(2 k-1) !} \int_{0}^{t}\left(\psi^{(2 k)}(0)-\psi^{(2 k)}(t)\right)(t-u)^{2 k-1} \mathrm{~d} u .
\end{aligned}
$$

Our aim now is to prove that the integral

$$
\int_{0}^{\delta_{0}} t^{-\alpha-1}\left|\Delta_{M}(t)\right| \mathrm{d} t
$$

converges. To this end, we notice that by virtue of (20) and (21)

$$
\begin{aligned}
\int_{0}^{\delta_{0}} t^{-\alpha-1}\left|\Delta_{M}(t)\right| \mathrm{d} t \leq & \frac{2}{(2 k-1) !} \int_{0}^{\delta_{0}} t^{\alpha-1} \int_{0}^{t}\left(\phi^{(2 k)}(0)-\phi^{(2 k)}(t)\right)(t-u)^{2 k-1} \mathrm{~d} u \mathrm{~d} t+\frac{2}{(2 k-1) !} \int_{0}^{\delta_{0}} t^{-\alpha-1} \\
& \times \int_{0}^{t}\left(\psi^{(2 k)}(0)-\psi^{(2 k)}(t)\right)(t-u)^{2 k-1} \mathrm{~d} u \mathrm{~d} t .
\end{aligned}
$$

Thus, it is sufficient to prove the convergence of two integrals on the right-hand side of (23). Let us prove this fact, for example, for the first one. We have

$$
\begin{aligned}
\int_{0}^{\delta_{0}} t^{-\alpha-1} \int_{0}^{t}\left(\phi^{(2 k)}(0)\right. & \left.-\phi^{(2 k)}(t)\right)(t-u)^{2 k-1} \mathrm{~d} u \mathrm{~d} t \\
\leq & \sum_{l=0}^{2 k-1} C_{2 k-1}^{l} \int_{0}^{\delta_{0}} t^{l-\alpha-1} \int_{0}^{t} u^{2 k-1-l}\left(\phi^{(2 k)}(0)-\phi^{(2 k)}(u)\right) \mathrm{d} u \mathrm{~d} t \\
= & \sum_{l=0}^{2 k-1} C_{2 k-1}^{l}\left(\left.\frac{t^{l-\alpha}}{l-\alpha} \int_{0}^{t} u^{2 k-1-l}\left(\phi^{(2 k)}(0)-\phi^{(2 k)}(u)\right) \mathrm{d} u\right|_{0} ^{\delta_{0}}\right. \\
& \left.-\frac{1}{l-\alpha} \int_{0}^{\delta_{0}} t^{-1-\lambda}\left(\phi^{(2 k)}(0)-\phi^{(2 k)}(t)\right) \mathrm{d} t\right) .
\end{aligned}
$$

Since the distribution corresponding to $\phi(t)$ possesses finite moment of $\alpha$ order and $\phi(t)$ is real-valued, we have according to Lemma 2.4

$$
\int_{0}^{\delta_{0}} u^{-1-\lambda}\left(\phi^{(2 k)}(0)-\phi^{(2 k)}(u)\right) \mathrm{d} u<\infty .
$$

For the same reason

$$
\lim _{t \rightarrow 0} t^{l-\alpha} \int_{0}^{t} u^{2 k-1-l}\left(\phi^{(2 k)}(0)-\phi^{(2 k)}(u)\right) \mathrm{d} u \leq \lim _{t \rightarrow 0} \int_{0}^{t} u^{-1-\lambda}\left(\phi^{(2 k)}(0)-\phi^{(2 k)}(u)\right) \mathrm{d} u=0 .
$$


Thus, the integral (22) converges and

$$
\int_{0}^{\delta_{0}} t^{-\alpha-1}\left|\Delta_{M}(t)\right| \mathrm{d} t<\infty
$$

Next, we define the function

$$
S_{M}(u)=\Delta_{M}\left(\mathrm{e}^{-u}\right)
$$

and rewrite (3) in the form

$$
q_{1} S_{M}\left(u+\gamma_{1}\right)+\ldots+q_{n-1} S_{M}\left(u+\gamma_{n-1}\right)+S_{M}(u)=0, \quad u \in(-\ln (\delta), \infty)
$$

where

$$
\gamma_{i}=-\ln \left(h_{i}\right)>0, \quad i=1, \ldots, n-1 .
$$

Without loss of generality we assume that $\delta=1$.

It follows from (24) that

$$
\int_{0}^{\infty}\left|S_{M}(u)\right| \mathrm{e}^{\alpha u} \mathrm{~d} u<\infty
$$

and Proposition 2.5 entails

$$
S_{M}(u) \equiv 0, \quad u>-\ln (\delta)
$$

Hence, $\Delta_{M}(t) \equiv 0$ that is $|f(t)|^{2}=|g(t)|^{2}$ for $t \in[0, \delta)$. Again we derive from Lemma 3.2 that there exists tending to zero sequence $s_{n}$ such that $\arg f\left(s_{n}\right)=\arg g\left(s_{n}\right)$ for all $n$. Since $|f(t)|$ and $|g(t)|$ do not vanish for $|t|<\delta$ and are equal there, we have

$$
f\left(s_{n}\right)=\left|f\left(s_{n}\right)\right| \mathrm{e}^{i \arg f\left(s_{n}\right)}=\left|g\left(s_{n}\right)\right| \mathrm{e}^{i \arg g\left(s_{n}\right)}=g\left(s_{n}\right)
$$

for sufficiently large natural number $n$ and Lemma 2.2 yields

$$
f^{(l)}(0)=g^{(l)}(0), \quad l=0, \ldots, 2 k .
$$

Let us choose $\delta>\delta_{1}>0$ in such a way that

$$
|f(t)-g(t)|<1 / 4, \quad|g(t)|>1 / 2
$$

for $|t|<\delta_{1}$. Expanding $\ln (x)$ in the Taylor series, we can write

$$
\Delta(t)=f(t)-g(t)+\frac{1-g(t)}{g(t)}(f(t)-g(t))-\sum_{j=2}^{\infty} \frac{(g(t)-f(t))^{j}}{j g^{j}(t)} .
$$

The Cauchy-Schwarz-Bunyakovskii inequality and (26) imply

$$
\int_{0}^{\delta_{1}} t^{-\lambda-1}\left|\frac{1-g(t)}{g(t)} \frac{(f(t)-g(t))}{t^{2 k}}\right| \mathrm{d} t<2\left(\int_{0}^{\delta_{1}} t^{-\lambda-1}|1-g(t)|^{2} \mathrm{~d} t\right)^{1 / 2}\left(\int_{0}^{\delta_{1}} t^{-\lambda-1}\left(\frac{f(t)-g(t)}{t^{2 k}}\right)^{2} \mathrm{~d} t\right)^{1 / 2}
$$

Due to Lemma 2.3

$$
|1-g(t)|^{2}=(1-\Re g(t))^{2}+(\Im g(t))^{2} \leq(1-\Re g(t))^{2}+2(1-\Re g(t)) \leq 3(1-\Re g(t)),
$$


and using Lemma 2.4 one has (it should be taken into account that the distribution $G(x)$ has finite absolute moment of $2 k+\lambda$ order)

$$
\int_{0}^{\delta_{1}} t^{-\lambda-1}|1-g(t)|^{2} \mathrm{~d} t \leq 3 \int_{0}^{\delta_{1}} t^{-\lambda-1}(1-\Re g(t)) \mathrm{d} t<\infty .
$$

Further,

$f(t)-g(t)=-\frac{1}{(2 k-1) !} \int_{0}^{t}\left(f^{(2 k)}(0)-f^{(2 k)}(t)\right)(t-u)^{2 k-1} \mathrm{~d} u+\frac{1}{(2 k-1) !} \int_{0}^{t}\left(g^{(2 k)}(0)-g^{(2 k)}(t)\right)(t-u)^{2 k-1} \mathrm{~d} u$ and

$$
\begin{aligned}
|f(t)-g(t)|^{2} \leq & \frac{2 t}{(2 k-1) !} \int_{0}^{t}\left|f^{(2 k)}(0)-f^{(2 k)}(t)\right|^{2}(t-u)^{4 k-2} \mathrm{~d} u \\
& +\frac{2 t}{(2 k-) !} \int_{0}^{t}\left|g^{(2 k)}(0)-g^{(2 k)}(t)\right|^{2}(t-u)^{4 k-2} \mathrm{~d} u .
\end{aligned}
$$

Making again use of the first inequality of Lemma 2.3 with regard to the fact that $\frac{f^{(2 k)}(t)}{f^{(2 k)}(0)}$ is characteristic function, we have

$$
\begin{aligned}
\left|f^{(2 k)}(0)-f^{(2 k)}(t)\right|^{2} & =\left(f^{(2 k)}(0)\right)^{2}\left|1-\frac{f^{(2 k)}(t)}{f^{(2 k)}(0)}\right|^{2}=\left(f^{(2 k)}(0)\right)^{2}\left(1-\Re \frac{f^{(2 k)}(t)}{f^{(2 k)}(0)}\right)^{2}+\left(f^{(2 k)}(0)\right)^{2}\left(\Im \frac{f^{(2 k)}(t)}{f^{(2 k)}(0)}\right)^{2} \\
& \leq 3\left(f^{(2 k)}(0)\right)^{2}\left(1-\Re \frac{f^{(2 k)}(t)}{f^{(2 k)}(0)}\right)
\end{aligned}
$$

Analogously

According to Lemma 2.4

$$
\left|g^{(2 k)}(0)-g^{(2 k)}(t)\right|^{2} \leq 3\left(g^{(2 k)}(0)\right)^{2}\left(1-\Re \frac{g^{(2 k)}(t)}{g^{(2 k)}(0)}\right)
$$

and

$$
\int_{0}^{\delta_{1}} t^{-1-\lambda}\left(1-\Re \frac{f^{(2 k)}(t)}{f^{(2 k)}(0)}\right) \mathrm{d} t<\infty
$$

$$
\int_{0}^{\delta_{1}} t^{-1-\lambda}\left(1-\Re \frac{g^{(2 k)}(t)}{g^{(2 k)}(0)}\right) \mathrm{d} t<\infty .
$$

Combining two last inequalities with $(30,31)$ and $(32)$, we derive

$$
\int_{0}^{\delta_{1}} t^{-\lambda-1}\left|\frac{f(t)-g(t)}{t^{2 k}}\right|^{2} \mathrm{~d} t<\infty .
$$

Further, by virtue of $(26)$

$$
\int_{0}^{\delta_{1}} t^{-\lambda-1}\left|\frac{f(t)-g(t)}{g(t)}\right|^{j} \mathrm{~d} t \leq \frac{4}{2^{j-2}} \int_{0}^{\delta_{1}} t^{-\lambda-1}|f(t)-g(t)|^{2} \mathrm{~d} t
$$

and using (33), one get

$$
\left|\int_{0}^{\delta_{1}} \sum_{j=2}^{\infty} \frac{(g(t)-f(t))^{j}}{j g^{j}(t)} \mathrm{d} t\right| \leq C \int_{0}^{\delta_{1}} t^{-\lambda-1}|f(t)-g(t)|^{2} \mathrm{~d} t<\infty .
$$


Upon setting

$$
\tilde{\Delta}(t)=\frac{\Delta(t)}{t^{2 k}}, \quad 0<|t|<\delta_{1},
$$

we get from the last inequality and inequalities $(27-29,33)$

$$
\tilde{\Delta}(t)=\frac{f(t)-g(t)}{t^{2 k}}+R(t), \quad 0<|t|<\delta_{1}
$$

where

Now the finiteness of the integral

$$
\int_{0}^{\delta_{1}} t^{-\lambda-1}|R(t)| \mathrm{d} t<\infty
$$

$$
\int_{0}^{\delta_{1}} t^{-\lambda-1}|\tilde{\Delta}(a t)+\tilde{\Delta}(b t)+\tilde{\Delta}(-(a+b) t)| \mathrm{d} t
$$

for any $0<a<1,0<b<1$ is equivalent to the finiteness of the integral

$$
\int_{0}^{\delta_{1}} t^{-2 k-\lambda-1}\left|\frac{f(a t)-g(a t)}{a^{2 k}}+\frac{f(b t)-g(b t)}{b^{2 k}}+\frac{\overline{f((a+b) t)}-\overline{g((a+b) t)}}{(a+b)^{2 k}}\right| \mathrm{d} t
$$

which, in its turn, is less than the sum of the two

$$
\begin{array}{r}
\int_{0}^{\delta_{1}} t^{-2 k-\lambda-1} \frac{1}{(2 k-1) !} \int_{0}^{t} \mid f^{(2 k)}(0)-f^{(2 k)}(a t)+f^{(2 k)}(0)-f^{(2 k)}(b t) \\
\quad+f^{(2 k)}(0)-f^{(2 k)}(-(a+b) t) \mid(t-u)^{2 k-1} \mathrm{~d} u \\
\times \int_{0}^{\delta_{1}} t^{-2 k-\lambda-1} \frac{1}{(2 k-1) !} \int_{0}^{t} \mid g^{(2 k)}(0)-g^{(2 k)}(a t)+g^{(2 k)}(0)-g^{(2 k)}(b t) \\
\quad+g^{(2 k)}(0)-g^{(2 k)}(-(a+b) t) \mid(t-u)^{2 k-1} \mathrm{~d} u
\end{array}
$$

Let us prove now that the both are finite. We have, for example, for the first one

$$
\begin{array}{r}
\int_{0}^{\delta_{1}} t^{-2 k-\lambda-1} \frac{1}{(2 k-1) !} \int_{0}^{t}\left(f^{(2 k)}(0)-\Re f^{(2 k)}(a t)+f^{(2 k)}(0)\right. \\
\left.-\Re f^{(2 k)}(b t)+f^{(2 k)}(0)-\Re f^{(2 k)}(-(a+b) t)\right)(t-u)^{2 k-1} \mathrm{~d} u \\
\quad+\int_{0}^{\delta_{1}} t^{-2 k-\lambda-1} \frac{1}{(2 k-1) !} \int_{0}^{t} \mid \Im f^{(2 k)}(a t)+\Im f^{(2 k)}(b t) \\
+\Im f^{(2 k)}(-(a+b) t) \mid(t-u)^{2 k-1} \mathrm{~d} u .
\end{array}
$$

Lemma 2.3, being applied to the characteristic function $\frac{f^{(2 k)}(t)}{f^{(2 k)}(0)}$, yields

$$
\begin{aligned}
&\left|\Im f^{(2 k)}(a t)+\Im f^{(2 k)}(b t)+\Im f^{(2 k)}(-(a+b) t)\right| \\
& \leq f^{(2 k)}(0)-\Re f^{(2 k)}(a t)+f^{(2 k)}(0)-\Re f^{(2 k)}(b t)+f^{(2 k)}(0)-\Re f^{(2 k)}(-(a+b) t) .
\end{aligned}
$$


Now the finiteness of the first integral in (34) follows from the finiteness of the integral

$$
\int_{0}^{\delta_{1}} t^{-2 k-\lambda-1} \int_{0}^{t}\left(f^{(2 k)}(0)-\Re f^{(2 k)}(t)\right)(t-u)^{2 k-1} \mathrm{~d} u .
$$

Finiteness of the second integral in (34) can be proved analogously. Thus, it is proved that

$$
\int_{0}^{\delta_{1}} t^{-\lambda-1}|\tilde{\Delta}(a t)+\tilde{\Delta}(b t)+\tilde{\Delta}(-(a+b) t)| \mathrm{d} t<\infty .
$$

Further, the real-valued characteristic function (as has been proved above $\Re \tilde{\Delta}(t)=0$ )

$$
N(t)=-i(\tilde{\Delta}(a t)+\tilde{\Delta}(b t)+\tilde{\Delta}(-(a+b) t))
$$

satisfies the equation

$$
q_{1} h_{1}^{2 k} N\left(h_{1} t\right)+\ldots+q_{n-1} h_{n-1}^{2 k} N\left(h_{n-1} t\right)+N(t)=0, \quad|t|<\delta
$$

for any $0<a<1$ and $0<b<1$. We also notice that $\lambda=\max \{\Re z: \tilde{\tau}(z)=0\}$, where

$$
\tilde{\tau}(z)=1+q_{1} h_{1}^{2 k+z}+\ldots+q_{n-1} h_{n-1}^{2 k+z} .
$$

Upon setting

$$
\tilde{S}(u)=N\left(\mathrm{e}^{-u}\right)
$$

we see that $\tilde{S}(u)$ satisfies the equation

$$
q_{1} \tilde{S}\left(u+2 k \gamma_{1}\right)+\ldots+q_{n-1} \tilde{S}\left(u+2 k \gamma_{n-1}\right)+\tilde{S}(u)=0, \quad u \in(-\ln (\delta), \infty),
$$

and (follows from (35))

$$
\int_{0}^{\infty} \mathrm{e}^{\lambda u}|\tilde{S}(u)| \mathrm{d} u<\infty
$$

Proposition 2.5 implies that $\tilde{S}(u) \equiv 0$ and therefore $N(t)=0$ for $|t|<\delta$. Thus,

$$
\tilde{\Delta}(a t)+\tilde{\Delta}(b t)+\tilde{\Delta}(-(a+b) t)=0, \quad 0<|t|<\delta, 0<a, b<1
$$

and

that is

$$
\tilde{\Delta}(t) \equiv c t, \quad c \in \mathbb{C},
$$

on $(-\delta, \delta)$ and due to $(25) c=0$.

$$
\Delta(t) \equiv c t^{2 k+1}
$$

Proof of Theorem 1.1. The condition $L_{\mathbf{X}} \cong L_{\mathbf{Y}}$ means in terms of $f_{1}(t)$ and $f_{2}(t)$, that

$$
f_{1}^{k_{1}}\left(b_{1} t\right) \cdot \ldots \cdot f_{1}^{k_{n}}\left(b_{n} t\right)=f_{2}^{k_{1}}\left(b_{1} t\right) \cdot \ldots \cdot f_{2}^{k_{n}}\left(b_{n} t\right), \quad t \in \mathbb{R} .
$$

Letting $f(t)=f_{1}(t)$ and $g(t)=f_{2}(t)$, we come to (12) and Theorem 3.1 can be applied. Let us consider now two functions

$$
f_{10}(t)=\exp \left[-|t|^{\mu}-A|t|^{\alpha}\left(1+B_{1} \mathrm{e}^{i \frac{t}{|t|} \beta \ln (|t|)}+D \ln ^{m}|t|\right)\right]
$$


and

$$
f_{20}(t)=\exp \left[-|t|^{\mu}-A|t|^{\alpha}\left(1-B_{2} \mathrm{e}^{i \frac{t}{|t|} \beta \ln (|t|)}+D \ln ^{m}|t|\right)\right]
$$

where $z=\alpha+i \beta$ is one of the roots of $\tau(z)$ and $\mu$ is taken in the following way: if $\alpha \leq 1$ we set $\mu=\alpha$, if $\alpha \geq 2$ then $\mu=2$. According to Lemma 2.8 these two functions are characteristic functions, $A, D, m \leq 2$ being appropriately chosen, for enough small $B_{1}, B_{2}$. If $B_{1} \neq B_{2}$, then for any sequence $t_{k} \rightarrow 0$ such that

$$
\cos \left(\beta \ln \left(t_{k}\right)\right) \neq 0, \quad k \in \mathbb{N}
$$

one has

$$
f_{10}\left(t_{k}\right) \neq f_{20}\left(t_{k}\right), \quad k \in \mathbb{N} .
$$

It is not difficult to show that for any $\epsilon>0$ and finite $\delta>0$

$$
\begin{aligned}
& \int_{0}^{\delta} t^{-\lambda-1+\epsilon}\left(1-\Re \frac{f_{0}^{(2 k)}(t)}{f_{0}^{(2 k)}(0)}\right) \mathrm{d} t<\infty, \\
& \int_{0}^{\delta} t^{-\lambda-1+\epsilon}\left(1-\Re \frac{g_{0}^{(2 k)}(t)}{g_{0}^{(2 k)}(0)}\right) \mathrm{d} t<\infty .
\end{aligned}
$$

According to Lemma 1.4, the corresponding distributions possess finite absolute moments of $\alpha-\epsilon$ order for $0<\epsilon<\alpha$. Moreover $f_{10}(t)$ and $f_{20}(t)$ satisfy the equation (30) if $\alpha$ is chosen as described above.

Proof of Theorem 1.4. The condition of independence of $L_{\mathbf{X}}$ and $L_{\mathbf{Y}}$ can be expressed in terms of $f(t, s), f_{1}(t)$ and $f_{2}(s)$ in such a way

$$
f_{1}^{k_{1}}\left(b_{1} t\right) \cdot \ldots \cdot f_{1}^{k_{n}}\left(b_{n} t\right) f_{2}^{k_{1}}\left(b_{1} s\right) \cdot \ldots \cdot f_{2}^{k_{n}}\left(b_{n} s\right)=f^{k_{1}}\left(b_{1} t, b_{1} s\right) \cdot \ldots \cdot f^{k_{n}}\left(b_{n} t, b_{n} s\right), \quad t, s \in \mathbb{R} .
$$

For any $0<\rho \leq 1$ let us set $f(t)=f(t, \rho t)$ and $g(t)=f_{1}(t) f_{2}(\rho t)$.

Now we are going to prove that the distribution corresponding to $f(t)$ (and also the one corresponding to $g(t)$ ) has finite absolute moment of the order $m$ if $F(x, y)$ has all absolute moments of the order $m$.

Indeed, for any two random variables $X_{1}$ and $X_{2}$ with the joint distribution function $F(x, y)$ we have

$$
\mathbf{E}\left|X_{1}+\rho X_{2}\right|^{m} \leq \sum_{k=0}^{m} C_{m}^{k} \rho^{m-k} \mathbf{E}\left|X_{1}\right|^{k}\left|X_{2}\right|^{m-k}<\infty .
$$

Theorem 3.1 entails

$$
f(t, \rho t)=f_{1}(t) f_{2}(\rho t), \quad|t|<\delta, \quad 0<\rho \leq 1
$$

that means

$$
f(t, s)=f_{1}(t) f_{2}(s), \quad|s| \leq|t|<\delta .
$$

One can prove in a similar way that

$$
f(t, s)=f_{1}(t) f_{2}(s), \quad|t| \leq|s|<\delta .
$$

Thus,

Let us consider now the function

$$
f(t, s)=f_{1}(t) f_{2}(s), \quad \max \{|t|,|s|\}<\delta .
$$

$$
\begin{aligned}
f_{0}(t, s)=\exp \left[-|t|^{\mu}-A_{1}|t|^{\alpha}\left(1+D_{1} \ln ^{m}|t|\right)-|s|^{\mu}-A_{2}|s|^{\alpha}\left(1+D_{2} \ln ^{m}|s|\right)\right. & \left.-B|t|^{\alpha / 2}|s|^{\alpha / 2 i \frac{t}{2|t|} \beta \ln (|t|)} \mathrm{e}^{i \frac{s}{2|s|} \beta \ln (|s|)}\right],
\end{aligned}
$$


where $z=\alpha+i \beta$ is one of the roots of $\tau(z)$ and $\mu$ is taken as follows: if $\alpha \leq 1$ we set $\mu=\alpha$,if $\alpha \geq 2$ then $\mu=2$. According to Lemma 2.7 this function is a characteristic function, $A, D, \bar{m} \leq 2$ being appropriately chosen. If $B \neq 0$, then for any sequences $t_{k} \rightarrow 0$ and $s_{k} \rightarrow 0$ such that

$$
\begin{gathered}
\cos \left(\frac{\beta}{2} \ln \left(t_{k}\right)\right) \neq 0, \quad \cos \left(\frac{\beta}{2} \ln \left(s_{k}\right)\right) \neq 0, \quad k \in \mathbb{N}, \\
f_{0}\left(t_{k}, s_{k}\right) \neq f_{10}\left(t_{k}\right) f_{20}\left(s_{k}\right), \quad k \in \mathbb{N} .
\end{gathered}
$$

It is also not difficult to demonstrate that $f_{0}(t, s)$ satisfies the equation (31) under such choice of $\alpha$. Moreover the distribution corresponding to $f_{0}(t, s)$ has all absolute moments of $\alpha-\epsilon$ order. Indeed, $f_{0}(t, 0)$ and $f_{0}(0, s)$ satisfy the condition of Lemma 2.4 and therefore $\mathbf{E}|X|^{\alpha-\epsilon}<\infty, \mathbf{E}|Y|^{\alpha-\epsilon}<\infty$ for any $\epsilon>0$. According to Holder's inequality

$$
\mathbf{E}|X|^{\alpha_{1}-\epsilon_{1}}|Y|^{\alpha_{2}-\epsilon_{2}} \leq\left(\mathbf{E}|X|^{\alpha-\epsilon}\right)^{r}\left(\mathbf{E}|Y|^{\alpha-\epsilon} \mid\right)^{s}<\infty
$$

where

and

$$
\alpha_{1}+\alpha_{2}=\alpha, \quad \epsilon_{1}+\epsilon_{2}=\epsilon
$$

$$
r=\frac{\alpha_{1}-\epsilon_{1}}{\alpha-\epsilon}, \quad s=\frac{\alpha_{2}-\epsilon_{2}}{\alpha-\epsilon}
$$

I am grateful to Professor S. Albeverio for his kind hospitality during my stay at the Institute für Mathematik Universität Bonn where this work was done. I thank also anonymous referee for very helpful comments.

\section{REFERENCES}

[1] D.B. Belomestny, To the problem of reconstructing the distribution of summands by the distribution of their sum. Theory Probab. Appl. 46 (2001).

[2] M. Krein, Sur le problème du prolongement des fonctions hermitiennes positives et continues. (French) C. R. (Doklady) Acad. Sci. URSS (N.S.) 26 (1940) 17-22.

[3] T. Kawata, Fourier analysis in probability theory. Academic Press, New York and London (1972).

[4] B.Ja. Levin, Distribution of zeros of entire functions. American Mathematical Society, Providence, R.I. (1964) viii+493 pp.

[5] Yu.V. Linnik, Linear forms and statistical criteria. I, II. (Russian) Ukrain. Mat. Žurnal 5 (1953) 207-243, 247-290.

[6] I. Marcinkiewicz, Sur une propriété de la loi de Gauss. Mat. Z. 44 (1938) 622-638.

[7] V.V. Petrov, Limit theorems of probability theory. Sequences of independent random variables. Oxford Science Publications. The Clarendon Press, Oxford University Press, New York, Oxford Stud. Probab. 4 (1995) xii+292 pp.

[8] A.V. Prohorov and N.G. Ushakov, On the problem of reconstructing the distribution of summands by the distribution of their sum. Theory Probab. Appl. 46 (2001).

[9] N.G. Ushakov, Selected topics in Characteristic functions. VSP, Utrecht and Tokyo (1999). 